\title{
METRIC SPACES ON WHICH CONTINUOUS FUNCTIONS ARE UNIFORMLY CONTINUOUS AND HAUSDORFF DISTANCE
}

\author{
GERALD BEER
}

\begin{abstract}
Atsuji has internally characterized those metric spaces $X$ for which each real-valued continuous function on $X$ is uniformly continuous as follows: (1) the set $X^{\prime}$ of limit points of $X$ is compact, and (2) for each $\varepsilon>0$, the set of points in $X$ whose distance from $X^{\prime}$ exceeds $\varepsilon$ is uniformly discrete. We obtain these new characterizations: (a) for each metric space $Y$, the Hausdorff metric on $C(X, Y)$, induced by a metric on $X \times Y$ compatible with the product uniformity, yields the topology of uniform convergence; (b) there exists a metric space $Y$ containing an arc for which the Hausdorff metric on $C(X, Y)$ yields the topology of uniform convergence; (c) the Hausdorff metric topology on $\mathrm{CL}(X)$ is at least as strong as the Vietoris topology. We also characterize those metric spaces whose hyperspace is such a space.
\end{abstract}

Let $\langle W, d\rangle$ be a metric space. If $K \subset W$ and $\varepsilon$ is positive, let $S_{\varepsilon}[K]$ denote the union of all open $\varepsilon$-balls whose centers run over $K$. If $K_{1}$ and $K_{2}$ are nonempty subsets of $W$, and for some $\varepsilon>0$ both $S_{\varepsilon}\left[K_{1}\right] \supset K_{2}$ and $S_{\varepsilon}\left[K_{2}\right] \supset K_{1}$, then the Hausdorff distance $h_{d}$ between them is given by the formula $h_{d}\left(K_{1}, K_{2}\right)=$ $\inf \left\{\varepsilon: S_{\varepsilon}\left[K_{1}\right] \supset K_{2}\right.$ and $\left.S_{\varepsilon}\left[K_{2}\right] \supset K_{1}\right\}$. Otherwise, we write $h_{d}\left(K_{1}, K_{2}\right)=\infty$. If we restrict $h_{d}$ to the closed nonempty subsets CL $(W)$ of $W$, then $h_{d}$ defines an infinite-valued metric. Basic facts about this metric can be found in Aubin [2], Castaing and Valadier [3], or Engelking [4]. Now let $C(X, Y)$ denote the continuous functions from a metric space $\left\langle X, d_{X}\right\rangle$ to a metric space $\left\langle Y, d_{Y}\right\rangle$. We denote uniform distance in $C(X, Y)$ by $d_{1}$, that is,

$$
d_{1}(f, g)=\sup \left\{d_{Y}(f(x), g(x)): x \in X\right\} .
$$

Alternatively, we can identify members of $C(X, Y)$ with their graphs in $X \times Y$, and define the distance between functions to be the Hausdorff distance between their graphs, as induced by some metric compatible with the product uniformity. For definiteness and computational simplicity, we choose the box metric:

$$
\rho\left[\left(x_{1}, y_{1}\right),\left(x_{2}, y_{2}\right)\right]=\max \left\{d_{X}\left(x_{1}, x_{2}\right), d_{Y}\left(y_{1}, y_{2}\right)\right\}
$$

In the sequel, whenever $f$ and $g$ are continuous, we shall write $d_{2}(f, g)$ for $h_{\rho}(f, g)$.

It is easy to check that $d_{2}(f, g) \leq d_{1}(f, g)$, so that the topology of uniform convergence is always at least as strong as the one defined by $d_{2}$. Furthermore, if $X$ is compact and $Y$ is arbitrary, then $d_{1}$ and $d_{2}$ are equivalent metrics on $C(X, Y)$ [7]. This equivalence has been the basis for numerous papers in approximation theory by Bl. Sendov, V. Popov, B. Penkov, V. Veselinov and their associates in Sofia (see, e.g, $\left[\mathbf{9}, 10\right.$ and 12]). However, $d_{1}$ and $d_{2}$ can be equivalent somewhat

Received by the editors December 14, 1984 and, in revised form, February 7, 1985.

1980 Mathematics Subject Classification. Primary 54B20; Secondary 54C35, 54E45.

Key words and phrases. Hausdorff metric, topology of uniform convergence, uniformly continuous function, function space, UC space, hyperspace, Vietoris topology. 
more generally. It is the main purpose of this article to characterize those spaces $X$ for which $d_{1}$ and $d_{2}$ define the same topologies on $C(X, Y)$, independent of the choice of target space $Y$. We shall show that the desired spaces $X$ are those for which

(1) the set $X^{\prime}$ of limit points of $X$ is compact,

(2) for each $\varepsilon>0$, the set $\left(S_{\varepsilon}\left[X^{\prime}\right]\right)^{c}$ is uniformly discrete, i.e., there exists $\delta>0$ such that whenever $x$ and $y$ are distinct points of $\left(S_{\varepsilon}\left[X^{\prime}\right]\right)^{c}$, then $d_{X}(x, y)>\delta$. Atsuji [1] has characterized such metric spaces in terms of their uniform properties in several ways, the most important of which was recently rediscovered by Hueber [5]: each real-valued continuous function on $X$ is uniformly continuous. In the sequel we shall call those metric spaces that exhibit properties (1) and (2) Atsuji spaces. Not surprisingly, these spaces are exactly those for which each open cover has a Lebesgue number. Although this is not hard to prove directly, it also follows from basic results regarding the fine uniformity on a paracompact space (see, e.g., $\S 36$ of [15]). Each Atsuji space is complete; in fact, Toader [13] has essentially observed that $X$ is an Atsuji space if and only if each sequence in $X$ with distinct terms such that pairs of terms are arbitrarily close frequently has a cluster point. However, Atsuji spaces need not be locally compact (but see [14]). For example, let $\left\{e_{n}: n \in Z^{+}\right\}$be the usual orthonormal basis in $l_{2}$, and let $X$ denote the subspace of $l_{2}$ consisting of the origin plus all points of the form $(1 / j) e_{n}$ with $\{n, j\} \subset Z^{+}$. We also mention that the problem of describing those metrizable topological spaces which admit an Atsuji metric has been considered by Nagata [8], Levshenko [6], and Rainwater $[\mathbf{1 1}]$.

We find it convenient to use a sequential characterization of Atsuji spaces slightly different from those given in $[\mathbf{1}, \mathbf{5}$, and $\mathbf{1 3}]$.

DEFINITION. A sequence $\left\{x_{n}\right\}$ of distinct terms in a metric space $\langle X, d\rangle$ is called a sequence of paired isolated points if for each $n$, the point $x_{n}$ is isolated in $X$, and $\lim _{n \rightarrow \infty} d\left(x_{2 n-1}, x_{2 n}\right)=0$.

The straightforward proof of the next lemma is omitted.

LEMMA. A metric space $\langle X, d\rangle$ is an Atsuji space if and only if each sequence of paired isolated points in $X$ has a cluster point, and each sequence in $X^{\prime}$ has a cluster point.

To show that the equivalence of $d_{1}$ and $d_{2}$ on $C(X, Y)$ forces $X$ to be an Atsuji space under most circumstances, we need a preliminary lemma. Recall that a subset $A$ of a set $B$ in a metric space is called $\delta$-dense in $B$ if $S_{\delta}[A] \supset B$.

LEMMA. Let $\left\langle X, d_{X}\right\rangle$ and $\left\langle Y, d_{Y}\right\rangle$ be metric spaces. Let $x \in X^{\prime}$, and let $\phi:[0,1]$ $\rightarrow Y$ be a path such that $\phi(0) \neq \phi(1)$. Then for each pair of positive numbers $\varepsilon$ and $\delta$ there exist $\{f, g\} \subset C(X, Y)$ such that

(i) both $g\left(S_{\varepsilon}[x]\right)$ and $f\left(S_{\varepsilon}[x]\right)$ are $\delta$-dense in $\phi([0,1])$;

(ii) $g(z)=f(z)=\phi(0)$ whenever $d_{X}(z, x) \geq \varepsilon$;

(iii) for every $z$, either $f(z)=\phi(0)$ or $g(z)=\phi(0)$.

PROOF. By the uniform continuity of $\phi$ there exists $n \in Z^{+}$such that $\{\phi(j / n)$ : $1 \leq j \leq n\}$ is $\delta$-dense in $\phi([0,1])$. Since $x$ is a limit point of $X$, we can find $2 n$ distinct points $\left\{x_{1}, \ldots, x_{n}, w_{1}, \ldots, w_{n}\right\}$ in $S_{\varepsilon}[x]$. Let $\left\{S_{\lambda_{j}}\left[x_{j}\right]: j=1, \ldots, n\right\} \cup$ $\left\{S_{\theta_{j}}\left[w_{j}\right]: j=1, \ldots, n\right\}$ be disjoint balls, each lying within $S_{\varepsilon}[x]$. The desired 
functions $f$ and $g$ may be defined by

$$
\begin{aligned}
& f(z)= \begin{cases}\phi\left[\frac{j}{n} \cdot\left(1-\frac{1}{\lambda_{j}} d_{X}\left(z, x_{j}\right)\right)\right] & \text { if } d_{X}\left(z, x_{j}\right)<\lambda_{j} \\
\phi(0) & \text { otherwise }\end{cases} \\
& g(z)= \begin{cases}\phi\left[\frac{j}{n} \cdot\left(1-\frac{1}{\theta_{j}} d_{X}\left(z, w_{j}\right)\right)\right] & \text { if } d_{X}\left(z, w_{j}\right)<\theta_{j}, \\
\phi(0) & \text { otherwise. }\end{cases}
\end{aligned}
$$

THEOREM 1. Let $\left\langle X, d_{X}\right\rangle$ be a metric space. The following are equivalent.

(a) $X$ is an Atsuji space;

(b) For each metric space $Y$ the Hausdorff metric on $C(X, Y)$ induced by the box metric on $X \times Y$ yields the topology of uniform convergence;

(c) There exists a metric space $Y$ containing an arc for which the Hausdorff metric on $C(X, Y)$ induced by the box metric on $X \times Y$ yields the topology of uniform convergence.

Proof. (a) $\rightarrow$ (b) By Theorem 1 of $[\mathbf{1}]$, each element of $C(X, Y)$ is uniformly continuous. Let $\left\{f_{n}\right\}$ be a sequence in $C(X, Y) d_{2}$-convergent to a continuous function $f$, and let $\varepsilon$ be positive. Choose $\delta>0$ such that whenever $d_{X}(x, z)<\delta$, then $d_{Y}(f(x), f(z))<\varepsilon / 2$. Let $\lambda=\min \{\varepsilon / 2, \delta\}$, and choose $N$ so large that $d_{2}\left(f_{n}, f\right)<$ $\lambda$ for all $n>N$. Fix $x$ and $n>N$, and pick $z$ satisfying $\rho\left[\left(x, f_{n}(x)\right),(z, f(z))\right]<\lambda$. In particular, $d_{X}(x, z)<\lambda \leq \delta$, and we obtain

$$
d_{Y}\left(f_{n}(x), f(x)\right) \leq d_{Y}\left(f_{n}(x), f(z)\right)+d_{Y}(f(z), f(x))<\lambda+\varepsilon / 2 \leq \varepsilon .
$$

It follows that $d_{1}\left(f_{n}, f\right) \leq \varepsilon$. This shows that $d_{2}$ is at least as strong as $d_{1}$. As noted earlier, we always have $d_{2} \leq d_{1}$.

(b) $\rightarrow$ (c) Trivial.

(c) $\rightarrow$ (a) Suppose that $d_{1}$ and $d_{2}$ define the same topology. We show that $X$ satisfies our sequential characterization of an Atsuji space. First, suppose that $\left\{x_{n}\right\}$ is a sequence of paired isolated points with no cluster point. Let $a$ and $b$ be distinct points of $Y$, and define $f: X \rightarrow Y$ by

$$
f(z)=\left\{\begin{array}{ll}
a & \text { if } z=x_{2 n} \\
b & \text { otherwise. }
\end{array} \text { for some } n,\right.
$$

For each $n \in Z^{+}$let $f_{n}$ agree with $f$ except at the points $x_{2 n-1}$ and $x_{2 n}$, where the values of $f$ are reversed; that is,

$$
f_{n}(z)= \begin{cases}a & \text { if } z=x_{2 n-1} \\ b & \text { if } z=x_{2 n} \\ f(z) & \text { otherwise. }\end{cases}
$$

Since $\left\{x_{n}\right\}$ has no cluster point, $f$ and $f_{n}$ are continuous. For each $n, d_{1}\left(f_{n}, f\right)=$ $d_{Y}(a, b)$, whereas $d_{2}\left(f_{n}, f\right) \leq d_{X}\left(x_{2 n-1}, x_{2 n}\right)$. Since $\left\{x_{n}\right\}$ is a sequence of paired isolated points, $\left\{f_{n}\right\} d_{2}$-converges to $f$. We have shown that $d_{1}$ and $d_{2}$ are not equivalent, a contradiction.

Second, suppose $\left\{x_{n}\right\}$ is a sequence of limit points of $X$ with no cluster point. For each $n$ choose $\varepsilon_{n}$ such that $0<\varepsilon_{n}<1 / 2 n$ and the collection $\left\{S_{\varepsilon_{n}}\left[x_{n}\right]: n \in Z^{+}\right\}$is pairwise disjoint. By assumption, there exists a continuous function $\phi:[0,1] \rightarrow Y$ for which $\phi(0) \neq \phi(1)$; denote $\phi(0)$ by $a$ and $\phi(1)$ by $b$. By the last lemma, for 
each $n \in Z^{+}$, there exist continuous functions $f_{n}$ and $g_{n}$ from $X$ to $Y$ such that (i) both $f_{n}\left(S_{\varepsilon_{n}}\left[x_{n}\right]\right)$ and $g_{n}\left(S_{\varepsilon_{n}}\left[x_{n}\right]\right)$ are $\varepsilon_{n}$-dense in $\phi([0,1])$, (ii) $g_{n}(z)=f_{n}(z)=a$ whenever $d_{X}\left(z, x_{n}\right) \geq \varepsilon_{n}$, (iii) $g_{n}(z) \neq a$ implies $f_{n}(z)=a$. Define $h: X \rightarrow Y$ by

$$
h(z)= \begin{cases}f_{n}(z) & \text { if for some } n, d_{X}\left(z, x_{n}\right)<\varepsilon_{n}, \\ a & \text { otherwise. }\end{cases}
$$

Since $\lim _{n \rightarrow \infty} \varepsilon_{n}=0$ and $\left\{x_{n}\right\}$ has no cluster point, the collection

$$
\left\{\overline{S_{\varepsilon_{n}}\left[x_{n}\right]}: n \in Z^{+}\right\} \cup\left\{\bigcap_{n=1}^{\infty}\left\{z: d_{X}\left(z, x_{n}\right) \geq \varepsilon_{n}\right\}\right\}
$$

is a locally finite closed cover of $X$. Since the restriction of $h$ to each set in the cover is continuous, the amalgamation is continuous [4]. For exactly the same reasons, for each $n \in Z^{+}$, the function $h_{n}: X \rightarrow Y$ defined by

$$
h_{n}(z)= \begin{cases}g_{n}(z) & \text { if } d_{X}\left(z, x_{n}\right)<\varepsilon_{n} \\ h(z) & \text { otherwise }\end{cases}
$$

is continuous. We claim that $d_{2}\left(h_{n}, h\right) \leq 1 / n$, more precisely, that $S_{1 / n}[h] \supset h_{n}$ and $S_{1 / n}\left[h_{n}\right] \supset h$. We verify just the first inclusion, leaving the second to the reader. If $d_{X}\left(z, x_{n}\right) \geq \varepsilon_{n}$, then $\left(z, h_{n}(z)\right)$ actually lies on the graph of $h$. If $d_{X}\left(z, x_{n}\right)<\varepsilon_{n}$, then by condition (i) $h_{n}(z)=g_{n}(z)$ lies in $\phi([0,1])$. Also by condition (i) there exists $w \in S_{\varepsilon_{n}}\left[x_{n}\right]$ for which $d_{Y}\left(f_{n}(w), g_{n}(z)\right)<\varepsilon_{n}$. As a result,

$$
\begin{aligned}
\rho\left[\left(z, h_{n}(z)\right),(w, h(w))\right] & =\rho\left[\left(z, g_{n}(z)\right),\left(w, f_{n}(w)\right)\right] \\
& <\max \left\{2 \varepsilon_{n}, \varepsilon_{n}\right\}<1 / n .
\end{aligned}
$$

Again, $\left(z, h_{n}(z)\right) \in S_{1 / n}[h]$, and it follows that $h_{n} \subset S_{1 / n}[h]$.

Finally, let $n$ be any integer satisfying $1 / n<\frac{1}{2} d_{Y}(a, b)$. By (i) there exists $z$ in $S_{\varepsilon_{n}}\left[x_{n}\right]$ for which $d_{Y}\left(g_{n}(z), b\right)<1 / n$. By condition (iii) we conclude that $f_{n}(z)=a$, whence $d_{Y}\left(h_{n}(z), h(z)\right)>\frac{1}{2} d_{Y}(a, b)$. Thus, although $\left\{h_{n}\right\} d_{2}$-converges to $h$, it does not uniformly converge to $h$.

It should be observed that we do need some additional assumption on $Y$ to guarantee that $C(X, Y)$ is sufficiently rich for there to be any hope that the equivalence of $d_{1}$ and $d_{2}$ on $C(X, Y)$ forces $X$ to be an Atsuji space. For example, if $Y$ is totally disconnected and $X$ is connected, then each element of $C(X, Y)$ is uniformly continuous, and Hausdorff distance in $C(X, Y)$ yields the topology of uniform convergence.

Atsuji spaces may also be characterized in terms of a relationship between the Hausdorff metric topology and the Vietoris topology [4] on the hyperspace CL $(X)$ of closed nonempty subsets of $X$.

THEOREM 2. The metric space $\langle X, d\rangle$ is an Atsuji space if and only if the Hausdorff metric topology on $\mathrm{CL}(X)$ is at least as strong as the Vietoris topology.

ProOF. We use yet another internal characterization of Atsuji spaces: $X$ is Atsuji if and only if whenever $A$ and $B$ are disjoint closed sets, there exists $\varepsilon>$ 0 for which $S_{\varepsilon}[A] \cap S_{\varepsilon}[B]=\varnothing[\mathbf{1}]$. Now a subbase for the Vietoris topology consists of all sets of the form $\Omega_{V}=\{A: A \in \mathrm{CL}(X), A \cap V \neq \varnothing\}$ and $\Sigma_{V}=$ $\{A: A \in \mathrm{CL}(X), A \subset V\}$ where $V$ is open in $X$. Suppose $X$ is an Atsuji space. Without this assumption, each $\Omega_{V}$ is $h_{d}$-open. If $A \in \Sigma_{V}$, choose $\varepsilon>0$ for which 
$S_{\varepsilon}[A] \cap S_{\varepsilon}\left[V^{c}\right]=\varnothing$. Clearly, $h_{d}(A, B)<\varepsilon$ implies $B \in \Sigma_{V}$. Conversely, suppose that the $h_{d^{-}}$-topology is at least as strong as the Vietoris topology. If $A$ and $B$ are disjoint closed sets, there exists $\delta>0$ for which $h_{d}(F, A)<\delta$ implies $F \in \Sigma_{B^{c}}$. Since $h_{d}\left(\overline{S_{\delta / 2}[A]}, A\right)<\delta$, we obtain $S_{\delta / 4}[A] \cap S_{\delta / 4}[B]=\varnothing$.

If $X$ is a complete metric space and $K(X)$ is the collection of nonempty compact subsets of $X$, then $K(X)$ is a closed subset of $\left\langle\mathrm{CL}(X), h_{d}\right\rangle[\mathbf{3}]$. One would expect that the collection of nonempty Atsuji subsets of $X$ also forms a closed subset of the hyperspace. This fails; in fact, for arbitrary $X$, the Atsuji subsets are dense in $\mathrm{CL}(X)$. To see this, let $F \in \mathrm{CL}(X)$ be arbitrary. By Zorn's Lemma, for each $\varepsilon>0$, the collection

$$
\{E: E \subset F \text { and } d(x, y) \geq \varepsilon \text { for each }\{x, y\} \subset E\}
$$

has a maximal element $E(\varepsilon)$. Since $E(\varepsilon)$ is uniformly discrete, $E(\varepsilon)$ is an Atsuji subset of $X$, and the maximality of $E(\varepsilon)$ yields $h_{d}(E(\varepsilon), F) \leq \varepsilon$.

Our last result answers a basic question: which metric spaces have Atsuji hyperspaces?

THEOREM 3. The hyperspace $\left\langle\mathrm{CL}(X), h_{d}\right\rangle$ is an Atsuji space if and only if either $X$ is compact or $X$ is uniformly discrete.

Proof. It is well known that $\left\langle\mathrm{CL}(X), h_{d}\right\rangle$ is compact whenever $X$ is compact [3]. If for some $\varepsilon>0$ we have $d\left(x_{1}, x_{2}\right)>\varepsilon$ whenever $\left\{x_{1}, x_{2}\right\} \subset X$, then $h_{d}\left(F_{1}, F_{2}\right)>\varepsilon$ whenever $\left\{F_{1}, F_{2}\right\} \subset \mathrm{CL}(X)$. In either case, $\mathrm{CL}(X)$ is an Atsuji space. To prove the converse, we assume that $\mathrm{CL}(X)$ is an Atsuji space, but $X$ is neither compact nor uniformly discrete, and reach a contradiction. Since $\mathrm{CL}(X)$ is an Atsuji space, and the singleton subsets of $X$ form a closed subset of the hyperspace, the metric space $X$ must be Atsuji. Since $X$ is not uniformly discrete, there is a point $p$ in $X^{\prime}$, and since $X$ is noncompact, there is a sequence $\left\{x_{n}\right\}$ in $X$ with no cluster point. For each $n \in Z^{+}$the set $\left\{x_{n}, p\right\}$ is a limit point of $\operatorname{CL}(X)$. However, the sequence $\left\{x_{1}, p\right\},\left\{x_{2}, p\right\},\left\{x_{3}, p\right\}, \ldots$ can have no cluster point in $\operatorname{CL}(X)$, or else $\left\{x_{n}\right\}$ would have a cluster point in $X$. This violates the compactness of the set of limit points of $\mathrm{CL}(X)$.

We remark in closing that Theorem 3 remains valid if we replace $\mathrm{CL}(X)$ by $K(X)$ in its statement.

\section{REFERENCES}

1. M. Atsuji, Uniform continuity of continuous functions of metric spaces, Pacific J. Math. 8 (1958), 11-16.

2. J. P. Aubin, Applied abstract analysis, Wiley, New York, 1977.

3. C. Castaing and M. Valadier, Convex analysis and measurable multifunctions, Springer-Verlag, Berlin, 1977.

4. R. Engelking, General topology, Polish Scientific Publishers, Warsaw, 1977.

5. H. Hueber, On uniform continuity and compactness in metric spaces, Amer. Math. Monthly 88 (1981), 204-205.

6. B. Levshenko, On the concept of compactness and point-finite coverings, Mat. Sb. 42 (1957), 479484.

7. S. Naimpally, Graph topology for function spaces, Trans. Amer. Math. Soc. 123 (1966), 267-272.

8. J. Nagata, On the uniform topology of bicompactifications, J. Inst. Polytech. Osaka City University 1 (1950), 28-38. 
9. B. Penkov and Bl. Sendov, Hausdorffsche metrik und approximationen, Numer. Math. 9 (1966), 214-226.

10. V. Popov and $\mathrm{Bl}$. Sendov, The exact asymptotic behavior of the best approximation by algebraic and trigonometric polynomials in the Hausdorff metric, Math. USSR-Sb. 18 (1972), 139-149.

11. J. Rainwater, Spaces whose finest uniformity is metric, Pacific J. Math. 9 (1959), 567-570.

12. Bl. Sendov, Certain questions in the theory of approximation of functions and sets in the Hausdorff metric, Russian Math. Surveys 24 (1969), 143-183.

13. Gh. Toader, On a problem of Nagata, Mathematica (Cluj) 20 (1978), 78-79.

14. W. Waterhouse, On UC spaces, Amer. Math. Monthly 72 (1965), 634-635.

15. S. Willard, General topology, Addison-Wesley, Reading, Mass., 1968.

Department of Mathematics, California State University, los Angeles, CALIFORNIA 90032 